\title{
Diffractive production of open charm and bottom at the LHC
}

\author{
Marta Łuszczak* ${ }^{*}$ \\ University of Rzeszów, PL-35-959 Rzeszów, Poland \\ E-mail: luszczak@univ.rzeszow.pl
}

\section{Rafał Maciuła}

Institute of Nuclear Physics PAN, PL-31-342 Cracow, Poland

E-mail: rafal.maciulaeifj.edu.pl

\section{Antoni Szczurek}

University of Rzeszów, PL-35-959 Rzeszów, Poland, and

Institute of Nuclear Physics PAN, PL-31-342 Cracow, Poland

E-mail: Antoni.Szczurek@ifj.edu.pl

\begin{abstract}
We discuss diffractive production of open charm and bottom mesons at the LHC. The differential cross sections for single- and central-diffractive mechanisms for $c \bar{c}$ and $b \bar{b}$ pairs are calculated in the framework of the Ingelman-Schlein model corrected for absorption effects to include Regge factorization breaking. In this approach one assumes that the pomeron has a well defined partonic structure, and that the hard process takes place in a pomeron-proton or proton-pomeron (single diffraction) or pomeron- pomeron (central diffraction) processes. Here, only the LO gluon-gluon fusion partonic subprocess is taken into consideration, which is calculated within simple collinear approximation. Both pomeron flux factors as well as parton distributions in the pomeron are taken from the $\mathrm{H} 1$ Collaboration analysis of diffractive structure function and diffractive dijets at HERA. The sub-leading corrections from reggeon type exchanges are explicitly calculated and also taken into consideration. Several quark-level differential distributions are shown. The hadronization of charm and bottom quarks is taken into account by means of fragmentation function technique. Predictions for single- and central-diffractive production in the case of inclusive $D$ and $B$ mesons, as well as $D \bar{D}$ correlations are presented, including detector acceptance of the ATLAS, CMS and LHCb Collaborations. The experimental aspects of possible standard and dedicated measurements are carefully discussed.
\end{abstract}

XXII International Workshop on Deep-Inelastic Scattering and Related Subjects

28 April - 2 May 2014

Warsaw, Poland

\footnotetext{
*Speaker.

†This work was supported by the Polish National Science Centre (on the basis of decision No. DEC2013/09/D/ST2/03724).
} 


\section{Introduction}

In this presentation we discuss diffractive processes (single and central) production of heavy quarks in the framework of Ingelman-Schlein model corrected for absorption. The formalism and more details has been shown and discussed elsewhere [2]. Such a model was used in the estimation of several diffractive processes $[3,4,5,6]$. The absorption corrections are necessary to understand a huge Regge-factorization breaking observed in single and central production at Tevatron. We mention also the QCD mechanism of diffractive dissociation of gluons into heavy quark pairs. However, the associated formalism and our results were discussed elsewhere [1]. We discuss predictions for single- and central-diffractive production in the case of inclusive production of $D$ and $B$ mesons. Also $D \bar{D}$ correlations are presented, including detector acceptance of the ATLAS, CMS and LHCb Collaborations.

\section{A sketch of formalism}

The mechanisms of the diffractive production of heavy quarks $(c \bar{c})$ discussed here are shown in Fig.1.

a)
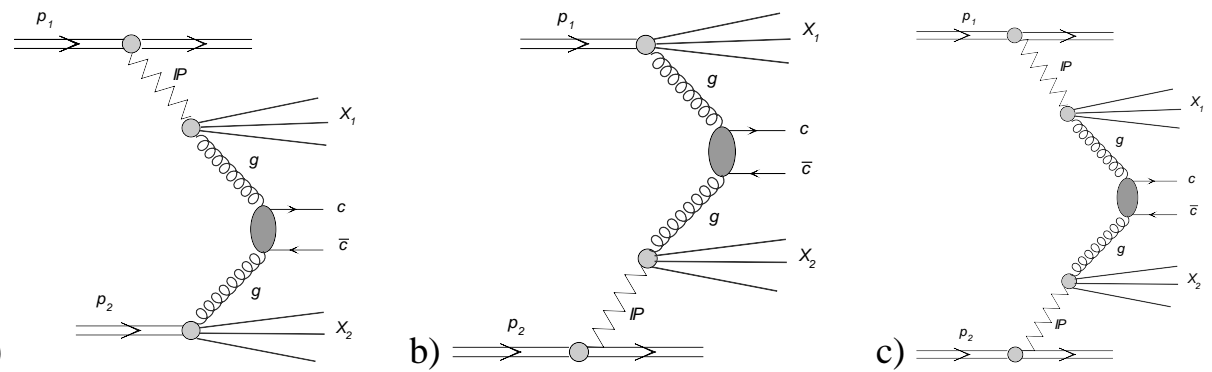

Figure 1: The mechanism of single-diffractive production of $c \bar{c}$ (panel a) and b) ) and mechanism of centraldiffractive production of heavy quarks (panel c))

In the following we apply the Ingelman and Schlein approach. In this approach one assumes that the Pomeron has a well defined partonic structure, and that the hard process takes place in a Pomeron-proton or proton-Pomeron (single diffraction) or Pomeron-Pomeron (central diffraction) processes. In this approach corresponding differential cross sections can be written as

$$
\begin{aligned}
& \frac{d \sigma_{S D}}{d y_{1} d y_{2} d p_{t}^{2}}=K \frac{\left|M_{g g \rightarrow Q \bar{Q}}\right|^{2}}{16 \pi^{2} \hat{s}^{2}}\left[\left(x_{1} q_{f}^{D}\left(x_{1}, \mu^{2}\right) x_{2} \bar{q}_{f}\left(x_{2}, \mu^{2}\right)\right)+\left(x_{1} \bar{q}_{f}^{D}\left(x_{1}, \mu^{2}\right) x_{2} q_{f}\left(x_{2}, \mu^{2}\right)\right)\right], \\
& \frac{d \sigma_{C D}}{d y_{1} d y_{2} d p_{t}^{2}}=K \frac{\left|M_{g g \rightarrow Q \bar{Q}}\right|^{2}}{16 \pi^{2} \hat{s}^{2}}\left[\left(x_{1} q_{f}^{D}\left(x_{1}, \mu^{2}\right) x_{2} \bar{q}_{f}^{D}\left(x_{2}, \mu^{2}\right)\right)+\left(x_{1} \bar{q}_{f}^{D}\left(x_{1}, \mu^{2}\right) x_{2} q_{f}^{D}\left(x_{2}, \mu^{2}\right)\right)\right]
\end{aligned}
$$

for single-diffractive and central-diffractive production, respectively. 
The 'diffractive' quark distribution of flavour $f$ can be obtained by a convolution of the flux of Pomerons $f_{\mathbf{I P}}\left(x_{\mathbf{I P}}\right)$ and the parton distribution in the Pomeron $q_{f / \mathbf{I P}}\left(\beta, \mu^{2}\right)$ :

$$
q_{f}^{D}\left(x, \mu^{2}\right)=\int d x_{\mathbf{I P}} d \beta \delta\left(x-x_{\mathbf{I P}} \beta\right) q_{f / \mathbf{P}}\left(\beta, \mu^{2}\right) f_{\mathbf{I P}}\left(x_{\mathbf{I P}}\right)=\int_{x}^{1} \frac{d x_{\mathbf{I P}}}{x_{\mathbf{I P}}} f_{\mathbf{I P}}\left(x_{\mathbf{I P}}\right) q_{f} / \mathbf{P} \mathbf{P}\left(\frac{x}{x_{\mathbf{I P}}}, \mu^{2}\right) .
$$

The flux of Pomerons $f_{\mathbf{I P}}\left(x_{\mathbf{I P}}\right)$ enters in the form integrated over four-momentum transfer

$$
f_{\mathbf{I P}}\left(x_{\mathbf{I P}}\right)=\int_{t_{\min }}^{t_{\max }} d t f\left(x_{\mathbf{I P}}, t\right)
$$

with $t_{\min }, t_{\max }$ being kinematic boundaries.

Both pomeron flux factors $f_{\mathbf{I P}}\left(x_{\mathbf{I P}}, t\right)$ as well as quark/antiquark distributions in the pomeron were taken from the $\mathrm{H} 1$ collaboration analysis of diffractive structure function and diffractive dijets at HERA [6]. The factorization scale for diffractive parton distributions is taken as $\mu^{2}=\hat{s}$.

In Ref.[1] we discussed in detail mechanism of diffractive dissociation of gluons into heavy quark pairs presented in Fig.2. There we presented relevant amplitudes, calculated relevant differential cross sections and compared to the Ingelman-Schlein model discussed here.
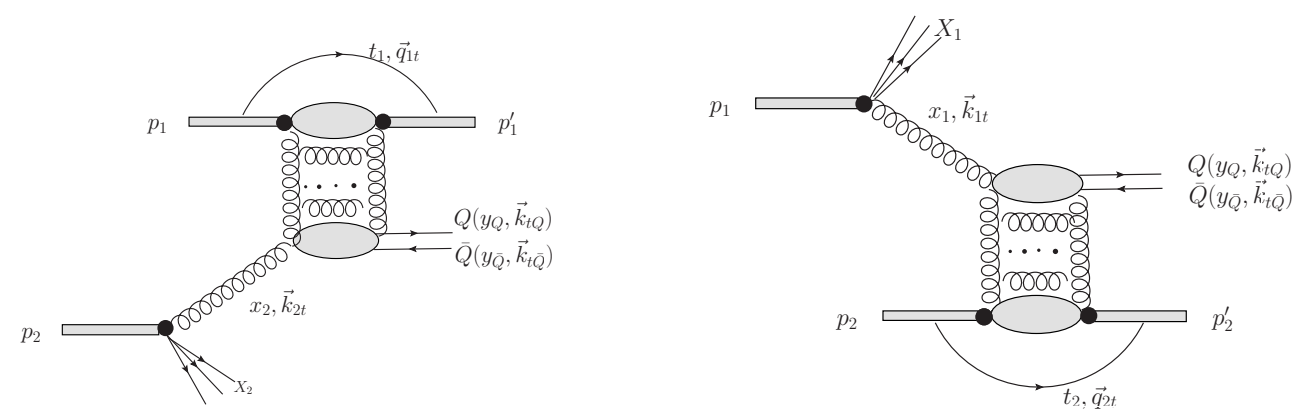

Figure 2: Diffractive dissociation of gluons into heavy quark pairs.

\section{Heavy quark hadronization effects}

The transition from quarks and gluons to hadrons, called hadronization or parton fragmentation, can be so far only approached through phenomenological models. In principle, in the case of many-particle final states the Lund string model [7] and the cluster fragmentation model [8] are usually used, giving description of the hadronization of the parton system as a whole. However, the hadronization of heavy quarks is usually done with the help of fragmentation functions (FFs) extracted from $e^{+} e^{-}$experiments (see e.g. Refs. [9, 10, 11]).

Especially in the case of diffractive production, where one or both protons remain intact, the applicability of the compound hadronization models (implemented in Monte Carlo generators and dedicated to non-diffractive processes) is still an open question. In our calculation we follow the fragmentation function technique which seems to be sufficient to illustrate the generic situation. 
This scheme has been recently successfully used for description of inclusive non-diffractive open charm and bottom data at the LHC [10,11]. In the context of diffractive production studies, the uncertainties coming from the process of parton fragmentation seem to be less important than those related to the parton-level diffractive calculation (e.g. uncertainties of diffractive PDFs or gap survival probability).

According to the fragmentation function formalism the differential distributions of open charm and bottom hadrons $h=D, B$, e.g. for single-diffractive production, are obtained through a convolution of differential distributions of heavy quarks/antiquarks and $Q \rightarrow h$ fragmentation functions:

$$
\left.\frac{d \sigma(p p \rightarrow h \bar{h} p X)}{d y_{h} d^{2} p_{t, h}} \approx \int_{0}^{1} \frac{d z}{z^{2}} D_{Q \rightarrow h}(z) \frac{d \sigma(p p \rightarrow Q \bar{Q} p X)}{d y_{Q} d^{2} p_{t, Q}}\right|_{\substack{y_{Q=y_{h}} \\ p_{t, Q}=p_{t, h} / z}}
$$

where $p_{t, Q}=\frac{p_{t, h}}{z}$ and $z$ is the fraction of longitudinal momentum of heavy quark $Q$ carried by hadron $h$. Since the rapidity spectra are usually flat, or slowly varying, the approximation assuming that $y_{Q}$ is unchanged in the fragmentation process, i.e. $y_{h}=y_{Q}$, is commonly applied. The fragmentation functions for heavy quarks are peaked at large $z$ (see Fig. 3) so the problematic small- $p_{t}$ region is suppressed.

In all the following numerical calculations the standard Peterson model of fragmentation function [12] is applied. The default set of the parameters for these functions is $\varepsilon_{c}=0.05$ for charm and $\varepsilon_{b}=0.004$ for bottom quarks. This values were extracted by H1 [13], ALEPH [14] and OPAL [15] analyses. In our calculation, to make the shapes of the Peterson functions closer to those from the FONLL approach, the parameters are fixed to $\varepsilon_{c}=0.02$ and $\varepsilon_{b}=0.001$ (see Fig. 3). In the following numerical predictions of the cross sections for $D^{0}$ and $B^{ \pm}$mesons the fragmentation functions are normalized to the branching fractions from Refs. [18, 19, 20], i.e. $\operatorname{BR}\left(c \rightarrow D^{0}\right)=0.565$ and $\mathrm{BR}\left(b \rightarrow B^{ \pm}\right)=0.4$.
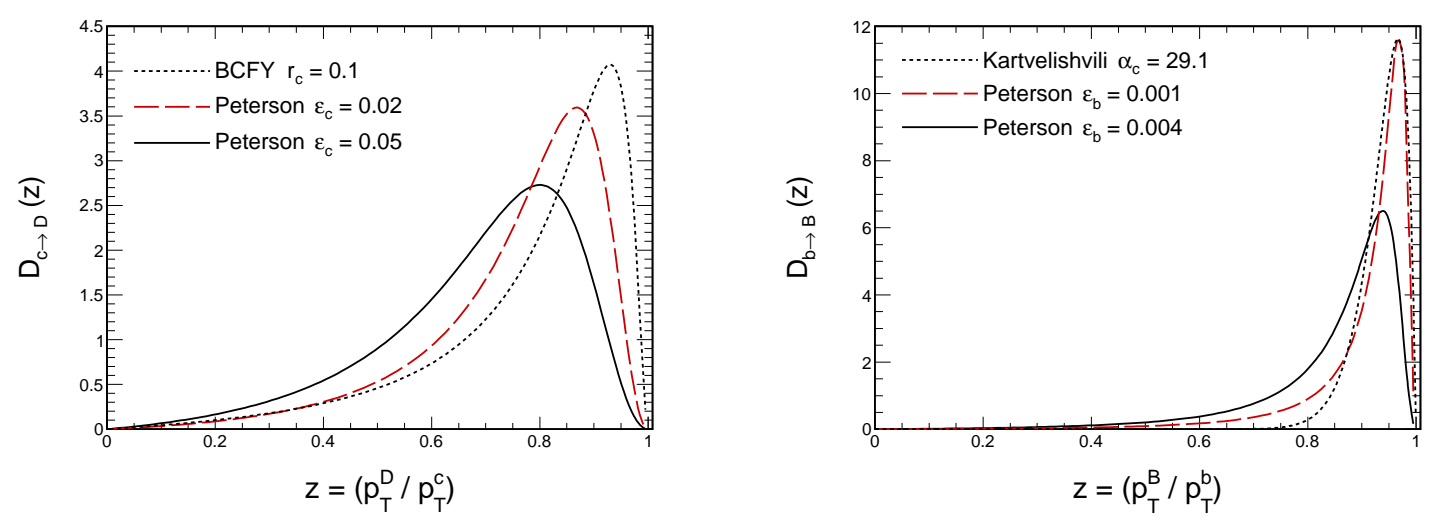

Figure 3: Different models of the fragmentation functions for charm (left) and bottom (right) quarks. The default functions from the FONLL framework are compared to the Peterson functions with different $\varepsilon$ parameters. 
Table 1: Integrated cross sections for diffractive production of open charm and bottom mesons in different modes for ATLAS, LHCb and CMS experiments at $\sqrt{s}=14 \mathrm{TeV}$.

\begin{tabular}{|c|c|c|c|c|}
\hline \multirow[b]{2}{*}{ Acceptance } & \multirow[b]{2}{*}{ Mode } & \multicolumn{3}{|c|}{ Integrated cross sections, [nb] } \\
\hline & & single-diffractive & central-diffractive & $\begin{array}{l}\text { non-diffractive } \\
\text { EXP data }\end{array}$ \\
\hline $\begin{array}{c}\text { ATLAS, }|y|<2.5 \\
p_{\perp}>3.5 \mathrm{GeV}\end{array}$ & $D^{0}+\overline{D^{0}}$ & 3461.88 (IR: $25 \%)$ & 68.45 (IR: 10\%) & - \\
\hline $\begin{array}{c}\mathrm{LHCb}, 2<y<4.5 \\
p_{\perp}<8 \mathrm{GeV}\end{array}$ & $D^{0}+\overline{D^{0}}$ & 42663.7 (IR: 29\%) & 766.5 (IR: 14\%) & $1488000 \pm 182000$ \\
\hline $\begin{array}{c}\text { CMS, }|y|<2.4 \\
p_{\perp}>5 \mathrm{GeV}\end{array}$ & $\left(B^{+}+B^{-}\right) / 2$ & $630.0(I R: 24 \%)$ & $11.74(I R: 9 \%)$ & $28100 \pm 2400 \pm 2000$ \\
\hline $\begin{array}{c}\mathrm{LHCb}, 2<y<4.5 \\
p_{\perp}<40 \mathrm{GeV}\end{array}$ & $B^{+}+B^{-}$ & $731.69(I R: 26 \%)$ & 11.85 (IR: 10\%) & $41400 \pm 1500 \pm 3100$ \\
\hline $\begin{array}{l}\mathrm{LHCb}, 2<y<4 \\
3<p_{\perp}<12 \mathrm{GeV}\end{array}$ & $D^{0} \overline{D^{0}}$ & $220.47(I R: 27 \%)$ & $3.68(I R: 11 \%)$ & $6230 \pm 120 \pm 230$ \\
\hline
\end{tabular}

\section{Numerical Results}

\subsection{Cross sections for $D^{0}$ and $B^{ \pm}$mesons production}

In Table 1 we present numerical predictions of the integrated cross sections for the single- and central-diffractive production of $D^{0}$ and $B^{ \pm}$mesons, including relevant experimental acceptance of the ATLAS, LHCb and CMS detectors.
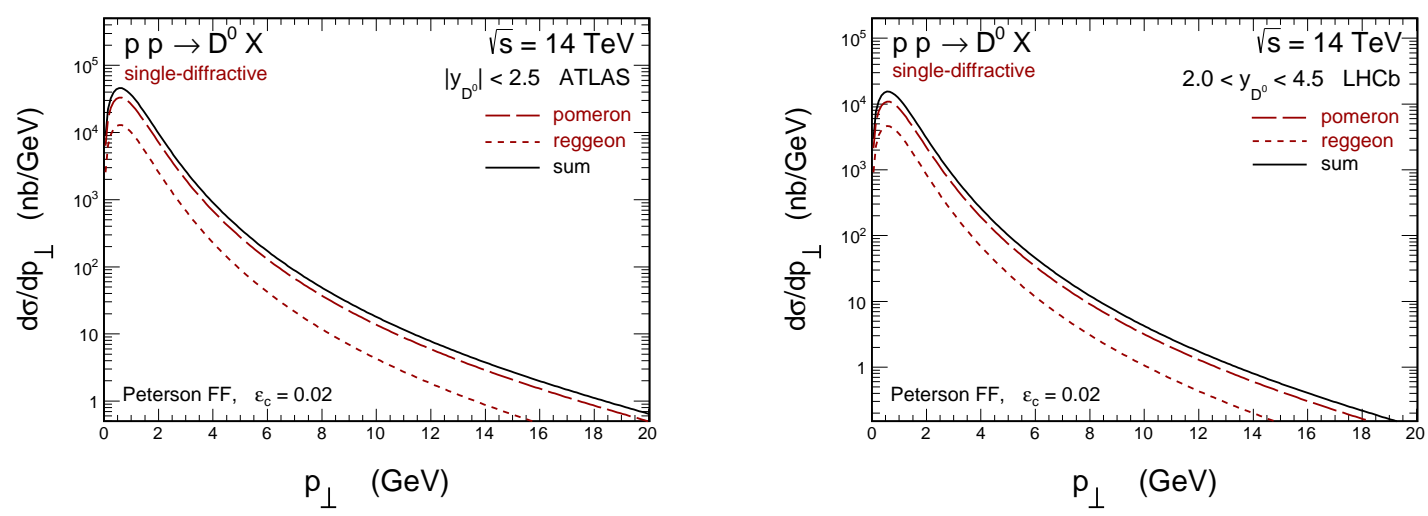

Figure 4: Transverse momentum distribution of $D^{0}$ meson within the ATLAS (left) and the LHCb (right) acceptance for single-diffractive production at $\sqrt{s}=14 \mathrm{TeV}$. Components of the Pomeron-gluon (and gluonPomeron) (long-dashed line) and the Reggeon-gluon (and gluon-Reggeon) (short-dashed line) mechanisms are shown separately.

Figures 4 and 5 show transverse momentum distributions of $D^{0}$ meson at $\sqrt{s}=14 \mathrm{TeV}$ within the ATLAS (left panels) and the LHCb (right panels) acceptance for single- and central-diffractive 
production respectively. The contributions of the Pomeron- (long-dashed lines) and Reggeonexchange (short-dashed lines) mechanisms are shown separately. These both contributions have similar shapes of the distributions and differ only in normalization. Therefore, considering this reaction one should not expect any possibility to extract and to test the Reggeon component within the special cuts in transverse momentum. The similar distributions with identical conclusions but for $B^{ \pm}$meson within the CMS (left panels) and the LHCb (right panels) acceptance are presented in Figs. 6 and 7.
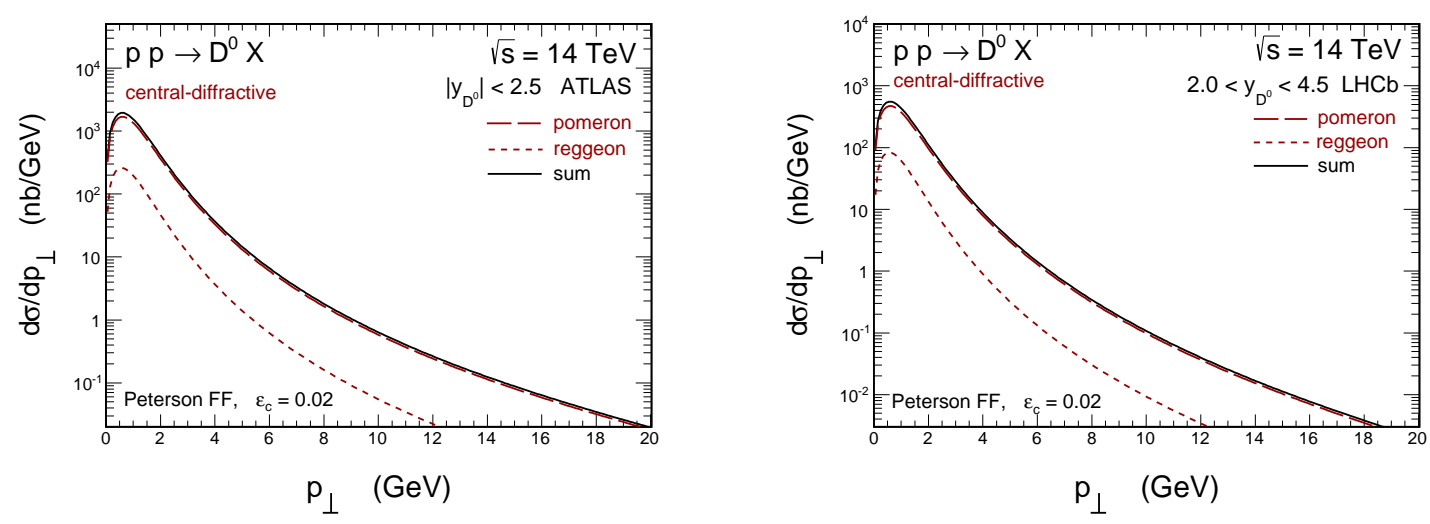

Figure 5: Transverse momentum distribution of $D^{0}$ meson within the ATLAS (left) and the LHCb (right) acceptance for central-diffractive production at $\sqrt{s}=14 \mathrm{TeV}$. Components of the Pomeron-Pomeron (longdashed line) and the Reggeon-Reggeon (short-dashed line) mechanisms are shown separately.
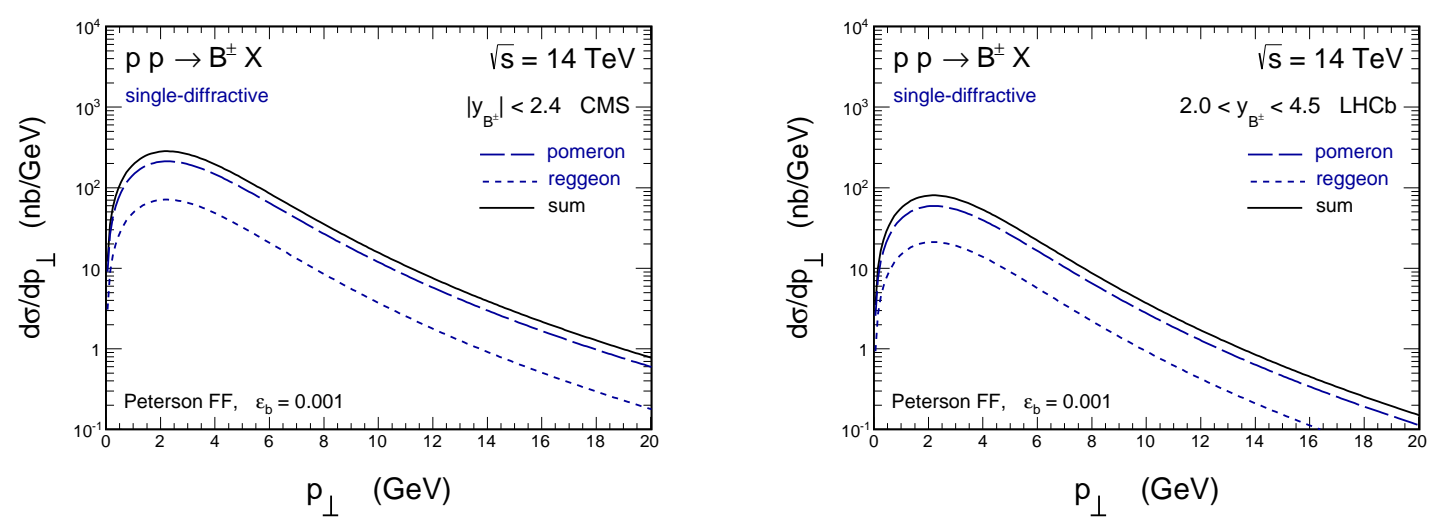

Figure 6: Transverse momentum distribution of $B^{ \pm}$meson within the CMS (left) and the LHCb (right) acceptance for single-diffractive production at $\sqrt{s}=14 \mathrm{TeV}$. Components of the Pomeron-gluon (and gluonPomeron) (long-dashed line) and the Reggeon-gluon (and gluon-Reggeon) (short-dashed line) mechanisms are shown separately.

\section{Conclusions}

We have calculated numerical predictions of the integrated cross sections for the single- and central-diffractive production of $D^{0}$ and $B^{ \pm}$mesons, including relevant experimental acceptance 

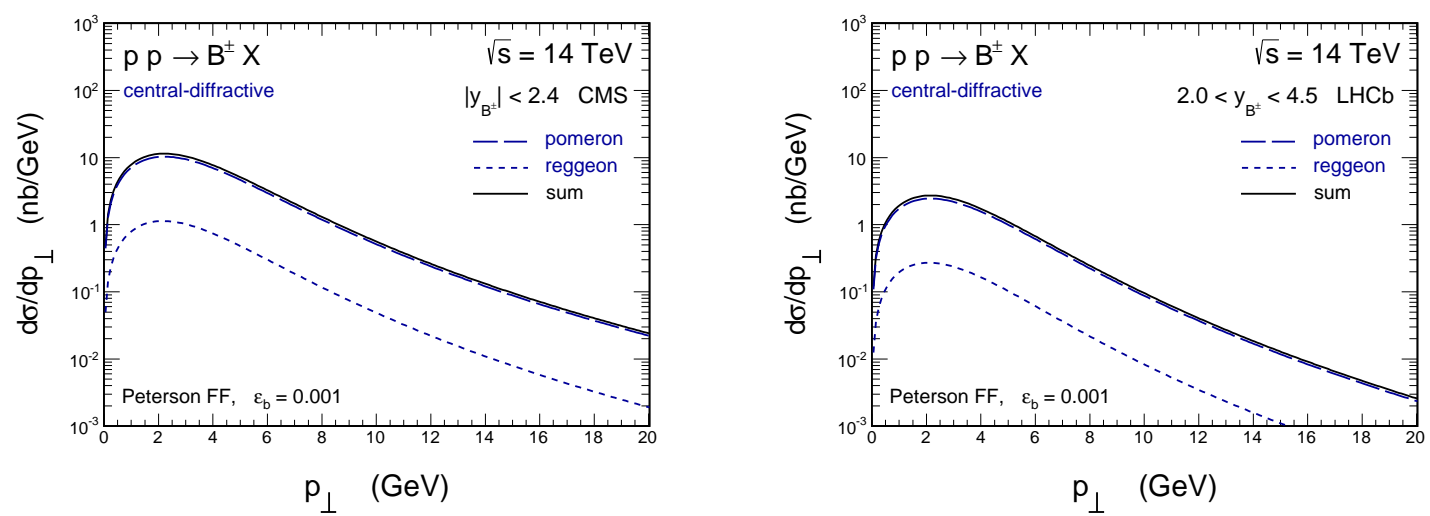

Figure 7: Transverse momentum distribution of $B^{ \pm}$meson within the CMS (left) and the LHCb (right) acceptance for central-diffractive production at $\sqrt{s}=14 \mathrm{TeV}$. Components of the Pomeron-Pomeron (longdashed line) and the Reggeon-Reggeon (short-dashed line) mechanisms are shown separately.

of the ATLAS, LHCb and CMS detectors. The kinematical cuts are taken to be identical to those which have been already used in the standard non-diffractive measurements of open charm and bottom production rates at the LHC. The corresponding experimental cross sections for non-diffractive processes are shown for reference. In the case of inclusive production of single $D$ or $B$ meson the ratio of the diffractive integrated cross sections to the non-diffractive one is of about $\sim 2 \%$ for single- and only about $\sim 0.05 \%$ for central-diffractive mechanism. This ratio is only slightly bigger for $D^{0} \overline{D^{0}}$ pair production, becoming $\sim 4 \%$ and $0.06 \%$, respectively. In addition, the relative contribution of the Reggeon-exchange mechanisms to the overall diffractive production cross sections is also shown. This relative $\frac{I R}{I P+I R}$ contribution is of about $\sim 25 \%$ for single- and $\sim 10 \%$ for central-diffractive processes for both, charm and bottom flavoured mesons. The ratio does not really change for different measurement modes and different experimental acceptance.

\section{References}

[1] M. Luszczak, W. Schafer, A. Szczurek, Phys. Lett. B 729, 15-23 (2014).

[2] M. Łuszczak, R. Maciuła and A. Szczurek, Phys. Rev. D84 (2011) 114018.

[3] G. Ingelman, P.E. Schlein, Phys.Lett.B152 (1985) 256.

[4] A. Berera, J.C. Collins, Nucl. Phys. B474 (1996) 183; R.J.M. Covolan and M.S. Soares, Phys. Rev. D67 (2003) 077504.

[5] M.V.T. Machado, Phys. Rev. D76 (2007) 054006.

[6] A. Aktas et al. [H1 Collaboration], Eur. Phys. J. C 48 (2006) 715.

[7] B. Andersson, G. Gustafson, G. Ingelman and T. Sjostrand, Phys. Rept. 97, 31 (1983).

[8] B. R. Webber, Nucl. Phys. B 238, 492 (1984).

[9] M. Cacciari, P. Nason and R. Vogt, Phys. Rev. Lett. 95, 122001 (2005) [hep-ph/0502203].

[10] M. Cacciari, S. Frixione, N. Houdeau, M. L. Mangano, P. Nason and G. Ridolfi, J. High Energy Phys. 10, 137 (2012) [arXiv:1205.6344 [hep-ph]]. 
[11] R. Maciula and A. Szczurek, Phys. Rev. D 87, 094022 (2013) [arXiv:1301.3033 [hep-ph]].

[12] C. Peterson, D. Schlatter, I. Schmitt and P. M. Zerwas, Phys. Rev. D 27, 105 (1983).

[13] F. D. Aaron et al. [H1 Collaboration], Eur. Phys. J. C 59, 589 (2009) [arXiv:0808.1003 [hep-ex]].

[14] A. Heister et al. [ALEPH Collaboration], Phys. Lett. B 512, 30 (2001) [hep-ex/0106051].

[15] G. Abbiendi et al. [OPAL Collaboration], Eur. Phys. J. C 29, 463 (2003) [hep-ex/0210031].

[16] E. Braaten, K. -m. Cheung, S. Fleming and T. C. Yuan, Phys. Rev. D 51, 4819 (1995) [hep-ph/9409316].

[17] V. G. Kartvelishvili, A. K. Likhoded and V. A. Petrov, Phys. Lett. B 78, 615 (1978).

[18] E. Lohrmann, arXiv:1112.3757 [hep-ex].

[19] J. Beringer et al. [Particle Data Group Collaboration], Phys. Rev. D 86, 010001 (2012).

[20] T. Aaltonen et al. [CDF Collaboration], Phys. Rev. D 77, 072003 (2008) [arXiv:0801.4375 [hep-ex]]. 